\title{
The Effect of a Simulation Education Program using Team- based Learning on Ego-Resilience, Communication, and Clinical Competence of Nursing Students
}

\author{
Hye Kyung $\mathrm{Oh}^{1}$ \\ ${ }^{1}$ Associate Professor, Department of Nursing, Daegu University, South Korea, kyungoh@daegu.ac.kr
}

\begin{abstract}
The team-based simulation education allows students to experience the process of evaluating and integrating new information and ideas through active interaction among team members in imitation of real-world scenario. This study investigated the effect of team-based simulation education program on ego-resilience, communication, and clinical competence among nursing students. This study was a quasi-experimental study, one-group pre-posttest design. The participants comprised 82 nursing students in their fourth year of college and a total of 20 teams of four to five members each were created. The team-based simulation education program in this study was conducted for two hours per week, for a total of 30 hours. All data were analyzed using SAS 9.2. The demographic characteristics, ego-resilience, communication, and clinical competence of participants were analysed using real numbers, percentages, and means. Ego-resilience, communication, and clinical competence according to the pre-and post-program were analysed using paired t-tests. The correlations among ego-resilience, communication, and clinical competence were measured used Pearson's correlation coefficients. The results of this study are as follows. The ego-resilience scores significantly increased after the intervention $(\mathrm{t}=2.84, \mathrm{p}=.0057)$. While communication scores increased after the intervention, no significant difference was found, $(\mathrm{t}=1.74, \mathrm{p}=.0859)$. Furthermore, the clinical competence scores for all five domains showed significant increases $(t=4.00, p=<.0001)$. A team-based simulation education program that replicates real-world clinical settings is believed to improve teamwork and enable students to acquire clinical competence and communication skills that can be applied to clinical practice. The program significantly improved ego-resilience, a psychological factor that influences practical competence, and this is significant in that this study proved that the program has a psychological effect in achieving quick and efficient psychological and physiological recovery using positive emotions in a stress situation.
\end{abstract}

Keywords: Simulation, Ego-Resilience, Communication, Clinical Competence

\section{Introduction}

\subsection{Necessity and Purpose}

The goal of nursing education is to cultivate clinically competent nursing professionals based on a scientific system of nursing knowledge[1]. Clinical competence refers to a combination of factorsself-awareness, motivation, emotional influence, skills, and knowledge - that enable nurses to provide quality care to patients and perform their clinical duties effectively. Clinical training offers nursing students the opportunity to care patients by practically integrating their theoretical knowledge.

Received: September 29, 2021; $1^{\text {st }}$ Review Result: November 15, 2021; $2^{\text {nd }}$ Review Result: December 31,2021 Accepted: January 29, 2022 
Furthermore, it improves their clinical competence through an integration and adjustment process by employing a holistic and comprehensive approach towards patients[2].

However, patients' increased rights to safety have made it difficult for nursing students to deliver care in clinical settings. This lack of opportunity forms a significant obstacle in attaining diverse clinical experiences[3]. In recent years, simulation education has been widely utilised as a teaching and learning strategy to address these shortcomings. Simulation education provides a safe learning environment and enables repeated practice for nursing students using highly computerised simulators[4].

Teaching and learning strategies that promote team learning activities and help students solve various problems that arise during teamwork are needed for simulation education. Thus, team-based learning can be used to ensure optimal learning outcomes.

Recent research on simulation education programs has focused on team learning. A study utilising a five-week, team-based simulation learning program among 63 third year nursing students reported improvements in interpersonal comprehension, problem-solving, knowledge, and clinical competence[5]. A further study comparing the scores for team performance among 229 fourth-year students before and after the nursing simulation program showed significantly higher scores in those who engaged in team-based learning[6].

Additionally, team-based learning allows students to experience the process of evaluating and integrating new information and ideas through active interaction among team members in imitation of real-world scenario[7]. Further, team-based learning can improve problem-solving and leadership by enhancing teamwork, improving communication among learners, and fostering critical thinking skills[8].

Clinical competence is crucial for nurses, and many nursing educational institutions strive to implement various teaching techniques, strengthen practicum, and develop and implement intervention programs to improve clinical competence. These strategies were developed based on the factors of nursing students such as satisfaction of major and clinical practice[9].

A recently emerged psychological factor, ego-resilience refers to the ability to flexibly respond to stressful situations by exercising self-control and involves the dynamic ability to maintain and enhance psychological balance.

Previous research indicates that ego-resilience is one of the most influential factors affecting the clinical competence of nursing students[9][10]. Nursing students are able to actively acquire clinical competence and improve ego-resilience while flexibly responding to reproduced clinical situations through simulation learning programs and by recognising and coping with adverse circumstances.

While numerous recent studies have examined the effects of simulation education on practical competence among nursing students, research examining the impact of these programs on egoresilience is rare. Furthermore, studies examining the effects of team-based simulation learning among nursing students are only recently emerging in Korea[5]. Additionally, studies that implemented teambased learning among medical, nursing, and dental hygiene students reported that students' learning achievements, problem-solving, and communication skills were improved[11].

Given team-based simulation practice training is needed because in practice, nursing work is performed in teams in clinical settings, the team-based simulation education in this study enhances the capability to perform nursing work by reproducing nursing cases with urgent and complex health problems. It was noted that previous studies on team-based simulation mostly analyzed and verified the effects on clinical practice competence. In previous studies, a very few studies analyzed the effect on ego-resilience, the ability to flexibly respond to stress situations originating from nursing patients with complex health problems. As beginners, the soon-to-graduate nursing students need egoresilience to cope with clinical stress situations and swiftly recover physically and psychologically with positive emotions and actions. This study thus aimed to verify the effect on ego-resilience, a 
psychological factor of nursing students, through team-based simulation education that allows them to directly experience complex patient nursing tasks, and to analyze the effect on the capabilities of communication and clinical competence required as part of clinical practice capabilities.

Thus, this study aimed to verify the effect on ego-resilience, a psychological factor of nursing students, though team-based simulation education that allows them to directly experience complex patient nursing tasks, and to analyze the effect on the capabilities of communication and clinical competence required as part of clinical practice capabilities.

\subsection{Hypothesis}

The hypotheses of this study investigated the effect of simulation education program using teambased learning on ego-resilience, communication, and clinical competence among nursing students. The hypotheses were as follows:

\subsubsection{First hypothesis}

Nursing students' ego-resilience will be increased after the simulation education program using team-based learning.

\subsubsection{Second hypothesis}

Nursing students' communication will be improved after the simulation education program using team-based learning.

\subsubsection{Third hypothesis}

Nursing students' clinical competence will be improved after the simulation education program using team-based learning.

\section{Theoretical Background}

Team-based learning can improve learners' problem-solving ability, interpersonal performance, practical knowledge, and clinical performance because they can experience the thinking process of evaluating, applying, and synthesizing new information and ideas through active interaction[12].

Simulation education is a useful learning method for nursing students to acquire nursing performance skills, including essential nursing skills, and also enhances learners' clinical performance ability, interpersonal understanding, and academic achievement[5]. Simulation practice training is an important opportunity to identify one's deficiencies, and clinical performance is trained since practical nursing interventions are performed in a direct manner[13].

In order for such simulation education to produce the best learning outcomes, team-based simulation education program will be conducive for nursing students' to developing clinical performance skills with which they should be equipped as future nurses. These programs will also contribute to improving the professional clinical performance required for the patient nursing with complex health problems, which would be difficult for nursing students to experience in person in hands-on clinical fields.

Excellent clinical competence is needed for individual nurses to better adapt to environments and perform professional nursing practice. Mitchell et al. (2012)[14] emphasized the importance of simple and clear-cut communication and posited that applying systematic communication can improve nurses' clinical competence.

Nurses mediate communication with many medical personnel and patients in the hospital, so they 
must be able to cope with various clinical situations and perform efficient communication, which contributes to minimizing possible safety accidents at clinical sites. Nurses can also ultimately improve the quality of nursing through the enhancement of both communication clarity and clinical competence by efficiently providing the information needed to report patient conditions in the process of communication with doctors or fellow nurses[15].

In a study by Lee \& Park[16], it was found that there was a positive correlation between egoresilience and clinical competence. A way to improve clinical competence in an unfamiliar hospital environment that nursing students have not experienced is thus to enhance the ego-resilience of nursing students.

Ego-resilience has been reported as an important factor in boosting clinical competence through adaptation to nursing studies and satisfaction with clinical practice[17].

As a result of reviewing the previous studies above, nursing students are anticipated to improve egoresilience by directly experiencing patient care and actively communicating with colleagues and other medical staff in a situation where complex patient cases are reproduced through team-based simulation education programs.

\section{Methods}

\subsection{Study Design}

This study employed a quasi-experimental, one-group pre-posttest design to analyze the effects of a simulation education program using team-based learning on ego-resilience, communication, and clinical competence among nursing students.

\subsection{Participants}

The participants comprised 82 nursing students in their fourth year of college. The sample size was determined using $G^{*}$ power 3.1 software. The required sample size for a significance $(\alpha)$ of .05 , power $(1-\beta)$ of .8 , and effect size (d) of .315 was 82 .

\subsection{Study Tools}

\subsubsection{Ego-Resilience}

Ego-resilience is an individual characteristic that manifests as the ability to view an internal or external problem with objective insight, restructure the situation, and flexibly respond[18]. In this study, ego-resilience was measured using a self-reported scale developed by Klohnen (1996)[18]and adapted by Park (1996)[19]. It comprises four subscales, including confidence, interpersonal efficiency, positive attitude, and emotional regulation. A total of 29 performance items were assessed for ego-resilience, and each item was scored from 1 to 5. Total scores ranged from 29 to 145, with higher scores indicating higher ego-resilience. In this study, the Cronbach's $\alpha$ was .906.

\subsubsection{Communication}

Communication skill refers to the ability to appropriately deliver one's opinions or clearly express one's views during a disagreement[20]. In this study, communication was measured using the Global Interpersonal Communication Competence Scale (GICC). This modified version[21] is based on the eight communication elements proposed in the Interpersonal Communication Competence Scale (ICC) developed by Rubin (1990)[20]. Each question is rated on a 5-point Likert scale, with total scores ranging from 15 to 75 . One assessor observed and evaluated these points, and higher scores indicated 
higher clinical competence. In this study, the Cronbach's alpha was $\alpha=.808$.

\subsubsection{Clinical Competence}

Clinical competence refers to the ability to proficiently perform one's roles in clinical practice based on appropriate knowledge, judgement, and skills. This study utilised Choi's version[22] ofClinical Competence Scale based on Schwirian's (1978)[23] Six Dimension Scale of Nursing Performance, which was adapted for nursing students. The questionnaire comprised a total of 45 items. Each item was rated on a 5-point Likert scale, with total scores ranging from 45 to 225 . Higher scores indicated greater clinical competence. In this study, the Cronbach's $\alpha$ was .938 .

\subsection{Simulation Education Program}

The simulation education program composed of 30 hours of learning for 15 weeks. Clinical situations simulated in the program were respiratory and gastrointestinal emergency patient care. Three to five students were randomly assigned to each group for simulation practice. The students were given the instruction of the simulated patient's room and devices, and each group was given an opportunity to practice using them. Each group used fifty minutes to discuss the patient's health problem, nursing care plan, role assignment for group members. Each group accomplishd the planned nursing care in the patient's room for 10-15minutes, followed by 15-20 minutes of reflection while watching the recorded group activities. After the completion of team-based activities, the entire class came together for 20 minutes of reflection study [Table 1].

[Table 1] Simulation Education Program using Team-based Learning

\begin{tabular}{|c|l|}
\hline Week & \multicolumn{1}{c|}{ Content } \\
\hline $1 \sim 2$ & $\begin{array}{l}\text { Orientation and pre-test } \\
\text { Team assign : 3 to 5 students per team } \\
\text { Instruction and practice about the simulated patient's room and devices }\end{array}$ \\
\hline $3 \sim 5$ & $\begin{array}{l}\text { Team discussion about disease for each case } \\
\text { Team discussion about core nursing skills for each case } \\
\text { Nursing skill practice and test }\end{array}$ \\
\hline $6 \sim 14$ & $\begin{array}{l}\text { Nursing care planning and presentation of each group } \\
\text { Simulation practice of each group } \\
\text { Reflective study and presentation of each group }\end{array}$ \\
\hline 15 & Evaluation and Post-test \\
\hline
\end{tabular}

\subsection{Data Collection}

Prior to the study, the participants signed a consent form indicating their consent to participate. The form thoroughly outlined the purpose and procedure of the study. A research assistant distributed the questionnaire before and after the simulation education program, after providing verbal instructions. The participants were informed in advance that their participation was voluntary and that they had the right to withdraw at any time without penalty. The participants were also informed that all data would be used only for research purposes and that anonymity and confidentiality would be guaranteed. Further, it was explained that all study-related records would be stored for three years upon completion of the study, after which they would be discarded. To ensure the ethical protection of the study participants, this study was approved by the Institutional Review Board at Daegu University (IRB acceptance number: 1040621-201804-HR-021-02). 
Groups were assigned such that each group comprised members with varying conditions, according to the principles of team-based learning. First, the participants were divided into high, middle, and low groups according to their mean value of ego-resilience scores. The participants were subsequently assigned to groups such that each group had an even distribution of students with high, middle, and low scores on the ego-resilience questionnaire. A total of 20 goups of three to five members each were thus created. Based on the results of a previous study by $\operatorname{Kim}(2012)[5]$ that confirmed the positive effects of a team-based simulation education program, the program in this study was conducted for two hours per week, for a total of 30 hours over 15 weeks.

Each group was given 50 minutes of discussion time to design the content of nursing planning, and role sharing among the group members to analyze and solve the health problems for respiratory and gastrointestinal emergency patient nursing cases. Nursing performance was conducted for 10-15 minutes for each group. The groups watched a recorded video and engaged in reflective learning for 15 minutes. After the team activity, reflective learning involving all students occurred for 20 minutes. Data were collected from March to June 2018.

\subsection{Data Analysis}

All data were analysed using SAS 9.2. The demographic characteristics, ego-resilience, communication, and clinical competence of participants were analyzed using real numbers, percentages, and mean. Ego-resilience, communication, and clinical competence according to the prepost program were analyzed using paired t-tests. The correlations among ego-resilience, communication, and clinical competence were analyzed used Pearson's correlation coefficients.

\section{Results}

\subsection{Demographic Characteristics}

The demographic characteristics of the participants are shown in [Table 2]. Forty-two(51.22\%) participants answered under 21 years, while 27 participants $(32.93 \%)$ said 22 years. A total of 74 (90.24\%) participants were female, while 8 (9.76\%) were male. Fifty-six $(68.29 \%)$ participants responded having a positive personality, while 52 participants $(63.41 \%)$ said they adapt well to situations and environmental changes. Forty-eight (58.54\%) participants responded having good health condition, while thirty-one participants $(37.80 \%)$ said they have moderate health condition. A total of $64(78.05 \%)$ participants were moderately satisfied with their college life, while $12(14.63 \%)$ were highly satisfied. A total of $58(70.73 \%)$ participants were moderately satisfied with their nursing major, while $23(28.05 \%)$ were highly satisfied. A total of 56 students $(68.29 \%)$ responded being satisfied with their clinical training, while 25 (30.49\%) were highly satisfied.

[Table 2] Demographic Characteristics of Participants

\begin{tabular}{|c|c|c|}
\hline \multicolumn{2}{|c|}{ Variables } & $\mathrm{N}(\%)$ \\
\hline \multirow{3}{*}{ Age (years) } & $\leq 21$ & $42(51.22)$ \\
\cline { 2 - 3 } & 22 & $27(32.93)$ \\
\cline { 2 - 3 } & $\geq 23$ & $13(15.86)$ \\
\hline \multirow{2}{*}{ Gender } & Female & $74(90.24)$ \\
\hline Social Support & Male & $8(9.76)$ \\
\cline { 2 - 3 } & Parents & $48(58.54)$ \\
\hline
\end{tabular}




\begin{tabular}{|c|c|c|}
\hline & Same sex friend & $21(25.61)$ \\
\hline & Friend of the opposite sex & $7(8.54)$ \\
\hline & Others & $6(7.32)$ \\
\hline \multirow{3}{*}{ Personality type } & Positive & $56(68.29)$ \\
\hline & Moderate & $24(29.27)$ \\
\hline & Negative & $2(2.44)$ \\
\hline \multirow{3}{*}{$\begin{array}{c}\text { Adaptationof ChangedSituationor } \\
\text { Environment }\end{array}$} & High & $52(63.41)$ \\
\hline & Moderate & $27(32.93)$ \\
\hline & Low & $3(3.66)$ \\
\hline \multirow{3}{*}{ Health Condition } & Good & $48(58.54)$ \\
\hline & Moderate & $31(37.80)$ \\
\hline & Poor & $3(3.66)$ \\
\hline \multirow{3}{*}{$\begin{array}{l}\text { Satisfactionwith } \\
\text { College Life }\end{array}$} & High & $12(14.63)$ \\
\hline & Moderate & $64(78.05)$ \\
\hline & Low & $6(7.32)$ \\
\hline \multirow{3}{*}{$\begin{array}{l}\text { Satisfactionwith } \\
\text { Nursing }\end{array}$} & High & $23(28.05)$ \\
\hline & Moderate & $58(70.73)$ \\
\hline & Low & $1(1.22)$ \\
\hline \multirow{3}{*}{$\begin{array}{l}\text { Satisfactionwith } \\
\text { Clinical Practice }\end{array}$} & High & $25(30.49)$ \\
\hline & Moderate & $56(68.29)$ \\
\hline & Low & $1(1.22)$ \\
\hline
\end{tabular}

\subsection{Hypothesis Verification}

\subsubsection{First Hypothesis}

Nursing students' ego-resilience will be increased after the simulation education program using team-based learning.

The ego-resilience scores of the participants between pre and post program are shown in [Table 3]. Paired t-test was performed to examine changes in the participants' ego-resilience scores after the program, compared to the baseline. Ego-resilience scores significantly increased after the program compared to the baseline $(\mathrm{t}=2.84, \mathrm{p}=.0057)$. The scores for the interpersonal efficiency $(\mathrm{t}=2.83$, $\mathrm{p}=.0059)$ and confidence $(\mathrm{t}=2.49, \mathrm{p}=.0148)$ domains of ego-resilience also showed significant positive changes. However, while the scores for positive attitude $(\mathrm{t}=1.44, \mathrm{p}=.1542)$ and emotional regulation $(\mathrm{t}=1.11, \mathrm{p}=.2701)$ increased after the program, these changes were not significant. The first hypothesis was supported by the increase in ego-resilience after simulation education program using team-based learning.

\subsubsection{Second Hypothesis}

Nursing students' communication will be improved after the simulation education program using team-based learning.

The communication scores of the participants between pre and post program are shown in [Table 3]. Paired t-test was performed to examine whether communication scores changed after the program. Results indicated that the scores increased overall but not significantly $(t=1.74, p=.0859)$. The second hypothesis was supported by the improvement in communication after simulation education program 
The Effect of a Simulation Education Program using Team-based Learning on Ego-Resilience, Communication, and Clinical Competence of Nursing Students

using team-based learning.

\subsubsection{Third Hypothesis}

Nursing students' clinical competence will be improved after the simulation education program using team-based learning.

The clinical competence scores of the participants between pre and post program are shown in [Table 3].

The scores for nursing processes $(t=5.38, p=<.0001)$, nursing skills $(t=7.78, p=<.0001)$, education and relationship $(\mathrm{t}=5.05, \mathrm{p}=<.0001)$, personal relationship and communication $(\mathrm{t}=4.52, \mathrm{p}=<.0001)$, and professional development $(\mathrm{t}=4.05, \mathrm{p}=<.0001)$ domains of clinical competence and the overall clinical competence score $(\mathrm{t}=4.00, \mathrm{p}=<.0001)$ indicated significant increases after the program. The third hypothesis was supported by the improvement in clinical competence after simulation education program using team-based learning.

[Table 3] Degree of Ego-Resilience, Communication, and Clinical Competence

\begin{tabular}{|c|c|c|c|c|}
\hline \multirow{2}{*}{\multicolumn{2}{|c|}{ variable }} & Pre-test & Post-test & \multirow{2}{*}{$\mathrm{t}(\mathrm{p})$} \\
\hline & & $\mathrm{M} \pm \mathrm{SD}$ & $\mathrm{M} \pm \mathrm{SD}$ & \\
\hline \multirow{5}{*}{ Ego-resilience } & Positive attitude & $3.48 \pm 0.52$ & $3.54 \pm 0.55$ & $1.44(0.1542)$ \\
\hline & Interpersonal Efficiency & $3.36 \pm 0.60$ & $3.53 \pm 0.69$ & $2.83\left(0.0059^{* *}\right)$ \\
\hline & Confidence & $3.43 \pm 0.55$ & $3.56 \pm 0.62$ & $2.49\left(0.0148^{*}\right)$ \\
\hline & Emotional regulation & $3.43 \pm 0.81$ & $3.52 \pm 0.91$ & $1.11(0.2701)$ \\
\hline & Total & $3.43 \pm 0.47$ & $3.55 \pm 0.55$ & $2.84\left(0.0057^{* *}\right)$ \\
\hline \multicolumn{2}{|c|}{ Communication } & $3.65 \pm 0.49$ & $3.74 \pm 0.36$ & $1.74(0.0859)$ \\
\hline \multirow{6}{*}{$\begin{array}{c}\text { Clinical } \\
\text { Competence }\end{array}$} & Nursing Processes & $3.46 \pm 0.39$ & $3.73 \pm 0.47$ & $5.38(<.0001 * * *)$ \\
\hline & Nursing Skills & $3.48 \pm 0.45$ & $3.92 \pm 0.49$ & $7.78\left(<.0001^{* * *}\right)$ \\
\hline & Education/ Relationship & $3.45 \pm 0.43$ & $3.73 \pm 0.46$ & $5.05(<.0001 * * *)$ \\
\hline & $\begin{array}{l}\text { Personal relationship/ } \\
\text { Communication }\end{array}$ & $3.50 \pm 0.48$ & $3.77 \pm 0.56$ & $4.52(<.0001 * * *)$ \\
\hline & Professional Development & $3.46 \pm 0.41$ & $3.69 \pm 0.48$ & $4.05(<.0001 * * *)$ \\
\hline & total & $3.47 \pm 0.35$ & $3.77 \pm 0.43$ & $4.00\left(<.0001^{* * *}\right)$ \\
\hline
\end{tabular}

\subsection{The Correlation between Ego-resilience, Communication, and Clinical Competence}

The correlation between ego-resilience, communication, and clinical competence are shown in [Table 4].

There was a significant positive correlation between ego-resilience scores and communicaton scores $(\mathrm{r}=0.4607, \mathrm{p}=<.0001)$. There was a significant positive correlation between ego-resilience scores and clinical competence scores $(\mathrm{r}=0.2700, \mathrm{p}=.0142)$. Additionally, there was a significant positive correlation between the clinical competence scores and communication scores $(\mathrm{r}=0.3379, \mathrm{p}=.0019)$.

[Table 4] Correlation among Ego-Resilience, Communication, and Clinical Competence

\begin{tabular}{|c|c|c|c|}
\hline & Ego-Resilience & Communication & Clinical Competence \\
\hline Ego-Resilience & 1 & & \\
\hline Communication & $0.4607\left(<.0001^{* * *}\right)$ & 1 & \\
\hline Clinical Competence & $0.2700\left(.0142^{*}\right)$ & $0.3379\left(.0019^{* *}\right)$ & 1 \\
\hline
\end{tabular}




\section{Discussion}

This study investigated the effect of team-based simulation education program on ego-resilience, communication, and clinical competence among nursing students. Furthermore, this study endeavoured to provide foundational data for developing teaching strategies essential for equipping nursing professionals with excellent clinical competence.

The participants' ego-resilience scores increased from 3.43 at baseline to 3.55 after the program, and the change was statistically significant. Multiple investigative studies on ego-resilience among nursing students have been published in recent years. The ego-resilience score found in this study was consistent with that found byLee and Park (2013) (3.44)[2], and Park and Han(2011) (3.46)[24]. Among the domains of ego-resilience, the score for positive attitude was the highest (3.48), followed by confidence (3.43), emotional regulation (3.43), and interpersonal efficiency (3.36). This was consistent with the scores found in previous research[2][24].

In this study, all domains of ego-resilience improved after team-based simulation education. Specifically, the scores for confidence (3.56) and interpersonal efficiency (3.53) improved significantly. It is theorised that the interactions and decision-making processes involved in teambased learning in a real-world scenario were replicated in the simulation, thus enhancing the efficiency of relationships. Additionally, performing nursing interventions improved students' confidence in practice. Positive attitude and emotional regulation of ego-resilience got better after education than before, but there was no statistically significant difference. Since nursing skills are applied in communication and cooperation between team members by conducting team-based simulations, it is construed that each team member's postivie attitude and emotional regulation have not had a significant effect.

Clinical competence was significantly improved from 3.42 at baseline to 3.77 after the program. This is consistent with the results of the study conducted by Kim (2012)[5], where the team-based simulation learning group showed significant improvements in clinical competence compared to the case-based learning and nursing skills training group. Further, the results support previous findings that simulation-based education enhances nursing students' clinical competence[25].

Additionally, successfully acquiring theoretical knowledge does not necessarily translate to excellent practical competence. Thus, an educational curriculum that links fundamental skills with clinical practice is needed to help students apply theoretical concepts in the practical clinical setting.

The results of this study provide evidence for the effectiveness of team-based simulation education involving the replication of actual nursing cases. Specifically, the results support implementing learning scenarios in which students are encouraged to communicate and cooperate with other team members and perform nursing skills according to the priorities outlined in the nursing process. This can be utilised as a curriculum that comprehensively links students' fundamental theoretical and practical skills to achieve clinical competence.

In this study, after team-based simulation education, nursing students showed improved communication skills compared to the baseline. However, the result was not significant. This is consistent with previous research that investigated the effects of team-based simulation learning[26].

Communication is an essential skill for nursing professionals and is at the core of nursing education. Communication is the most influential therapeutic strategy and forms a crucial component in achieving positive health outcomes. Thus, fostering communication skills is emphasised in nursing education. However, nursing students lack opportunities to communicate with colleagues, other health professionals, and patients in the clinical setting.

Team-based learning is a process of achieving shared team goals through discussion and communication among members. Effective communication cannot be simply acquired by taking relevant courses. Rather, it must be attained via systematic learning coupled with practical application. 
Thus, a team-based simulation education program that provides real-world scenarios is a teaching strategy that enables students to engage in active communication with team members, patients, and other health professionals and review and improve health outcomes.

Currently, nursing students are faced with difficult circumstances where they cannot directly experience the patient nursing with urgent and complex health problems in hand-on clinical fields. Under this situation, it was identied that team-based simulation education effectively allows nursing students to experience and repeatedly learn various and complex patient care cases, thus improving ego-resilience to respond flexibly, communication with their colleagues and other medical staff, thus enhancing clinical competence.

\section{Conclusions}

This study aimed to investigate the effects of a team-based simulation education program on nursing students' ego-resilience, communication, and clinical competence.

A team-based simulation education program that replicates real-world clinical settings was tested in terms of improving teamwork and enabling students to acquire clinical competence and communication skills that can be applied to clinical practice. This is achieved by enhancing cooperative relationships with colleagues, patients, and other health professionals, communication, problem-solving, and nursing skills.

In this study, the team-based simulation education program significantly improved ego-resilience, a psychological factor that influences practical competence. Thus, this study proved that the program has a positive psychological effect on students in achieving quick and efficient psychological and physiological recovery using positive emotions in stressful situations.

In a clinical situation where nursing students need to solve complex nursing problems, team-based simulation education programs were identified to increase their ego-resilience and enhance communication and clinical competence. It can be seen that the team-based simulation education programs, which reproduce complex clinical situations that are difficult for nursing students to experience in person, are conducive to boosting both the psychological and practical capabilities of nursing students. This study had several limitations. The study was conducted with current students from only one nursing school; therefore, the results should be interpreted with caution. Replication studies are needed to substantiate these findings. There has thus far been a lack of research to measure the effect of team-based simulation education on the performance of the entire team. It is noted that this is due to the lack of research on the development of such tools, and that there are a few tools to measure the performance of the entire team. Therefore, it is recommended that there is a need to develop the tools to verify the degree of improvement of these team capabilities.

\section{Acknowledgments}

This work was supported by the 2018 Daegu University Research Grant under Grant 20180433.

\section{References}

[1] M. S. Lee, S. W. Hahn, Effect of simulation-based practice on clinical performance and problem solving process for nursing students, The Journal of Korean Academic Society of Nursing Education, (2011), Vol.17, No.2, pp.226-234, UCI: G704-001961.2011.17.2.006

[2] E. K. Lee, J. A. Park, Ego-resilience and the clinical competence of nursing students, Journal of Korean Public Health 
Nursing, (2013), Vol.27, No.2, pp.293-303, UCI: G704-SER000009448.2013.27.2.010

[3] M. N. Lee, K. D. Nam, H. Y. Kim, Effects of Simulation With Problem-Based Learning Program on Metacognition, Team Efficacy, and Learning Attitude in Nursing Students: Nursing Care With Increased Intracranial Pressure Patient, CIN: Computers, Informatics, Nursing, (2017), Vol.35, No.3, pp.145-151, DOI: https://doi.org/10.1097/CIN.0000000000000308

[4] M. A. Kaddoura, New Graduate Nurses' Perceptions of the Effects of Clinical Simulation on Their Critical Thinking, Learning, and Confidence, The Journal of Continuing Education in Nursing, (2010), Vol.41, No.11, pp.506-516, DOI: https://doi.org/10.3928/00220124-20100701-02

[5] H. R. Kim, Development and effect of team based simulation learning program on undergraduate nursing students, Chosun University, Ph.D dissertation, pp.53-71, (2012)

[6] Y. S. Roh, S. S. Kim, S. A. Park, J. W. Ahn, Effects of a Simulation With Team-Based Learning on Knowledge, Team Performance, and Teamwork for Nursing Students, CIN: Computers, Informatics, Nursing, (2020), Vol.38, No.7, pp.367-372, DOI: http://dx.doi.org/10.1097/CIN.0000000000000628

[7] P. G. Koles, A. Stolfi, N. J. Borges, S. Nelson, D. X. Parmelee, The Impact of Team-Based Learning on Medical Students' Academic Performance, Academic Medicine, (2010), Vol.85, No.11, pp.1739-1745, DOI: http://dx.doi.org/10.1097/ACM.0b013e3181f52bed

[8] H. A. Mennenga, T. Smyer, A Model for Easily Incorporating Team-Based Learning into Nursing Education, International Journal of Nursing Education Scholarship, (2010), Vol.7, No.1, pp.1-14, DOI: https://doi.org/10.2202/1548-923X.1924

[9] J. H. Cho, Factor influencing clinical competency of nursing students, Chosun University, Master's thesis, pp.17-24, (2014)

[10] Y. S. Park, Y. H. Kwon, Ego Resilience, Problem Solving Process and Clinical Practice Competency in Nursing Students, Journal of Korean Academic Society of Nursing Education, (2013), Vol.19, No.3, pp.341-350, DOI: http://dx.doi.org/10.5977/jkasne.2013.19.3.341

[11] A. R. Cho, S. I. Han, S. H. Yoon, J. H. Park, N. J. Yoo, S. Kim, Methods of Effective Team-Based Learning Administration and Expected Effects on Medical Education, Korean Journal of Medical Education, (2010), Vol.22, No.1, pp 47-55, DOI: http://dx.doi.org/10.3946/kjme.2010.22.1.47

[12] J. H. Seo, Impact of team based learning on nursing students' learning performance, Seonam University, Ph.D dissertation, pp.54-66, (2012)

[13] K. A. Kang, S. H. Kim, S. J. Kim, M. N. Lee, Content Analysis of Debriefing after Simulation-based Nursing Education on Respiratory Distress Syndrome in the Neonatal Intensive Care Unit, Child Health Nursing Research, (2018), Vol.24, No.2, pp.208-219, DOI: https://doi.org/10.4094/chnr.2018.24.2.208

[14] E. L. Mitchell, D. Y. Lee, S. Arora, K. L. Kwong, T. K. Liem, G. L. Landry, G. L. Moneta, N. Sevdalis, SBAR M\&M: A feasible, reliable, and valid tool to assess the quality of, surgical morbidity and mortality conference presentations, The American Journal of Surgery, (2012), Vol.203, No.1, pp.26-31, DOI: https://doi.org/10.1016/j.amjsurg.2011.07.008

[15] Y. H. Kim, Y. S. Choi, H. Y. Jun, M. J. Kim, Effects of SBAR Program on Communication Clarity, Clinical Competence and Self-efficacy for Nurses in Cancer Hospitals, The Korean Journal of Rehabilitation Nursing, (2016), Vol.19, No 1, pp.20-29, DOI: https://doi.org/10.7587/kjrehn.2016.20

[16] E. K. Lee, J. A. Park, Ego-Resilience and the Clinical Competence of Nursing Students, Journal of Korean Public Health Nursing, (2013), Vol.27, No.2, pp.293-303, DOI: https://doi.org/10.5932/JKPHN.2013.27.2.293

[17] Y. W. Shin, H. Lee, Y. Lim, Predictors of clinical competence in new graduate nurses, Journal of Korean Academy of Nursing Administration, (2010), Vol.16, No.1, pp.37-47, DOI: https://doi.org/10.11111/jkana.2010.16.1.37

[18] E. C. Klohnen, Conceptual analysis and measurement of the construct of ego-resiliency, Journal of personality and social psychology, (1996), Vol.70, No. 5, pp.1067-1079, DOI: http://dx.doi.org/10.1037/0022-3514.70.5.1067

[19] H. J. Park, Perceived stress, coping process and depression to ego-resilience, Korea University, Master's thesis, 
The Effect of a Simulation Education Program using Team-based Learning on Ego-Resilience, Communication, and Clinical Competence of Nursing Students

pp.10-25, (1996)

[20] R. B. Rubin, Communication competence, Speech Communication: Essay to Communication the 75th anniversary of the Speech Communication Association, Southern Illinois University Press, pp.94-129, (1990)

[21] K. H. Hur, Construction and Validation of a Global Interpersonal Communication Competence Scale, Korean Journal of Journalism \& Communication Studies, (2003), Vol.47, No.6, pp.380-408, UCI: G704000203.2003.47.6.003

[22] M. S. Choi, A study on the relationship between teaching effectiveness of clinical nursing education and clinical competency in nursing students, Ewha Woman's University, Master's thesis, pp.25-53, (2005)

[23] P. M. Schwirian, Evaluating the performance of nurses: a multidimensional approach, Nursing Research, (1978), Vol.27, No.6, pp.347-351, DOI: http://dx.doi.org/10.1097/00006199-197811000-00004

[24] W. J. Park, J. Y. Han, The Effect of Ego-resilience, Stress Coping Styles, Teaching, Effectiveness, and Family Support on Satisfaction of Clinical Practice in Nursing Students by AMOS Structural Equation Model, The Journal of Korean Academic Society of Nursing Education, (2011), Vol.17, No.3, pp.365-375, DOI: http://dx.doi.org/10.5977/JKASNE.2011.17.3.365

[25] S. O. Kim, S. Y. Pak, Effects of High-Fidelity Simulation-Based Training of Nursing Students according to their Learning Styles, The Journal of the Korea Contents Association, (2013), Vol.13, No.11, pp.1046-1057, DOI: http://dx.doi.org/10.5392/JKCA.2013.13.11.1046

[26] S. J. Park, S. J. Kim, The Effects of Team-based Simulation Education on Problem Solving Process, Health Communication, the Official Journal of Korean Academy on Communication in Healthcare, (2019), Vol.14, No.2, pp.165-172, DOI: http://dx.doi.org/10.15715/kjhcom.2019.14.2.165 